\title{
Hospital at Home and Emergence of the Home Hospitalist
}

\author{
Per Danielsson, MD*; Bruce Leff, MD²
}

${ }^{1}$ Hospital Medicine Program, Swedish Health Services, Seattle, Washington; 2Division of Geriatric Medicine and Gerontology, Johns Hopkins University School of Medicine, Baltimore, Maryland.

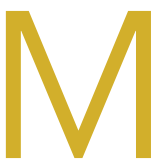

s. P., an 86-year-old woman with a history of hypertension, hyperlipidemia, coronary artery disease, and transient ischemic attack, presents to the emergency department with a three-day history of cough, fever, purulent sputum, fatigue, and dyspnea on exertion. Her vital signs are notable for a fever of $39.0^{\circ} \mathrm{C}$, blood pressure $136 / 92$, pulse 102 , respiratory rate 30 , and room air oxygen saturation of $91 \%$. She looks ill. She has a white blood cell count of 16,000, lactate 1.9, and a right lower lobe infiltrate on imaging. The emergency department attending physician presents the case to you for admission, and you accept the patient into your inpatient hospitalist service.

Now, let's imagine a different future in which you are the attending hospitalist on your institution's Hospital at Home $(\mathrm{HaH})$ service, where you will provide hospital-level care to Ms. P. in the comfort of her own home. Hospitalists should prepare for this paradigm shift.

\section{WHAT IS HOSPITAL AT HOME?}

$\mathrm{HaH}$ provides hospital-level care in a patient's home, for those with qualifying acute illnesses and appropriate degrees of acuity, as a substitute for traditional inpatient care. ${ }^{1}$ This is achieved by bringing the critical elements of hospital care to the home-physician and nursing care, intravenous medications and fluids, oxygen and respiratory therapies, basic radiography and ultrasound, durable medical equipment, skilled therapies, and more. ${ }^{2}$

All hospitalists have cared for patients like Ms. P., and she and many patients like her will have a straightforward hospital trajectory: initial evaluation in the emergency department, inpatient care provided by a hospitalist inpatient service, a few days of intravenous antibiotics and other hospital services, and finally, discharge to home.

\section{A SHARED RATIONALE FOR HOSPITAL MEDICINE AND HOSPITAL AT HOME}

However, not all patients will experience a smooth, or safe, hospital course. Studies that launched the hospital safety movement also provide the rationale for $\mathrm{HaH}$, namely, that hospitals are often dangerous environments for patients. ${ }^{3}$

*Corresponding Author: Per Danielsson, MD; E-mail: per.danielsson@swedish org; Telephone: 206-215-2370.

Published online first March 20, 2019.

Received: November 12, 2018; Revised: December 28, 2018;

Accepted: January 6, 2019

() 2019 Society of Hospital Medicine DOI 10.12788/jhm.3162
A complementary approach to improving outcomes for patients at high risk of iatrogenic illness such as functional decline, falls, delirium, adverse drug events, and hospital-associated disability syndrome, ${ }^{4-6}$ is to care for patients outside the traditional inpatient hospital environment. Over the past 20 years, many studies-including dozens of randomized controlled trials and several meta-analyses-have shown better outcomes for patients cared for in $\mathrm{HaH}$ : decreased length of stay, decreased incidence of adverse events (including substantially lower six-month mortality), better patient and caregiver care experiences, lower caregiver stress, and lower costs..$^{7-9} A$ recent Center for Medicare and Medicaid Innovation (CMMI) Demonstration conducted at the Mount Sinai Health System found similar results. ${ }^{10}$

\section{GROWING INTEREST IN HOSPITAL AT HOME AND CHALLENGES TO DISSEMINATION}

Interest in $\mathrm{HaH}$ has increased markedly over the past few years with increased penetration of Medicare and Medicaid managed care, the development and spread of accountable care organizations (ACOs), and a shift in focus among some health systems towards value-based care, population health, and community-based care. Recently, commercial entities have entered the $\mathrm{HaH}$ space and have raised substantial capital to fund development. Despite this growing interest in $\mathrm{HaH}$ and substantial evidence of its effectiveness, $\mathrm{HaH}$ has not been widely implemented or scaled in the United States.

Widespread dissemination and implementation of $\mathrm{HaH}$ has been hampered by several barriers. First, despite growing interest in $\mathrm{HaH}$, the culture of healthcare and health system leadership, for the most part, remains focused on facility-based care. ${ }^{11}$

Second, while $\mathrm{HaH}$ makes financial sense in the managed care arena, given the strong evidence for high-quality, lower-cost care, there is currently no standard payment mechanism for $\mathrm{HaH}$ in fee-for-service Medicare or in the commercial insurance space. However, there are indications that this may soon change. In the fall of 2017, a proposal for a bundled payment mechanism for acute $\mathrm{HaH}$ care plus 30 days of postacute care was unanimously approved by an Advisory Committee to the Secretary of the Department of Health and Human Services (HHS). ${ }^{12,13}$ The HHS Secretary recently noted that "the Department of Health and Human Services is keenly interested in ideas for home-based, hospital-level care, and agrees ... that this proposal holds promise for testing." 14

Third is the need to create the logistics and supply chain to support $\mathrm{HaH}$. There currently exists a well-established supply 
chain for providing hospital care. A hospitalist orders a dose of intravenous antibiotic or oxygen, and it is supplied in a timely manner. Similarly, the postacute sector of healthcare has a robust supply chain, though it operates on a somewhat different clock from the acute care setting. However, there is currently no easily replicable supply chain to meet the needs of providing acute care in the home. Each $\mathrm{HaH}$ has had to create its own system of logistics with the existing healthcare assets in its local environment. Developing this capacity at scale will require significant capital investment.

There are examples where $\mathrm{HaH}$ has scaled. Beginning in 1994, in the state of Victoria, Australia (population 6.3 million), the health authority reimbursed $\mathrm{HaH}$ care at the same rates as traditional hospital care. At last report, $\mathrm{HaH}$ provided approximately $5 \%$ of all hospital bed days of care in Victoria. Providing $\mathrm{HaH}$ on this scale helped avoid the need to build a new 500bed hospital to care for those patients. ${ }^{15}$ The avoided costs of building new hospital beds (and the ongoing need to fill those beds) represents significant societal return on investment attributable to $\mathrm{HaH}$.

\section{EMERGENCE OF THE HOME HOSPITALIST?}

A key element in implementing a HaH program is its physician staff in terms of the types of doctors who provide $\mathrm{HaH}$ care, how they are organized, and how they interact with patients. To date, $\mathrm{HaH}$ physicians have been predominantly geriatricians, but internists and family medicine physicians, employed as full-time members of a dedicated $\mathrm{HaH}$ team, also provide care by physically visiting patients in their homes. The reason for significant involvement of geriatricians in $\mathrm{HaH}$ may relate to the fact that geriatric fellowship training includes training in home-based medical care, whereas this is less common in family medicine and internal medicine residency training programs.

In order to provide $\mathrm{HaH}$ on a nationwide scale, there will be a need for a larger workforce. There is an opportunity here to leverage existing hospital physician staff, such as hospitalists. In addition, while there is significant value in physicians seeing patients in their homes, more scalable versions of $\mathrm{HaH}$ are being developed and implemented that leverage biometrically enhanced telemedicine approaches for a dedicated physician component of care, with in-person visits provided by other members of an interdisciplinary team.

We believe that hospitalists can play a key role as $\mathrm{HaH}$ physicians as the $\mathrm{HaH}$ model continues to evolve and expand. Hospitalists bring valuable expertise relevant to $\mathrm{HaH}$ care delivery, including extensive experience with the triage of acutely ill patients, an understanding of the natural course of acute illness and team-based care, and for some, experience with telemedicine care.

While a hospitalist providing $\mathrm{HaH}$ care would leverage many of the competencies of the traditional hospitalist, we suggest that such a provider should receive additional training and clinical experience in home-based medical care to help them better understand the unique aspects of providing care in patients' homes. ${ }^{16}$ Such training could include experience in mak- ing house calls, which can be a transformational experience in helping physicians improve their skills in dealing with social determinants of health, diagnosing and managing geriatric syndromes, and mobilizing community resources in the care of their patients, as well as managing care transitions. Hospitalists delivering care in $\mathrm{HaH}$ may also need to upgrade specific clinical skills commonly addressed by home-based medical care providers: wound care, caregiver-related issues, social and ethical issues specific to home-based care, problems with functional status, psychiatric and cognitive issues, management of gastrostomy tubes and bladder catheters, dermatologic problems, as well as palliative care and end-of-life symptom management. These skills are slightly different from the usual realm of the typical hospitalists' wheelhouse. However, it is all learnable. ${ }^{17}$ Similarly, geriatricians can learn from hospitalists as the $\mathrm{HaH}$ model evolves; there are $\mathrm{HaH}$ programs in existence today that take care of a sicker tranche of patients than earlier versions of $\mathrm{HaH}$, with continuous telemonitoring of patients and the ability to rapidly deploy providers, labs, imaging, and medications. Going forward, as healthcare organizations begin to develop HaH programs staffed by hospitalists, it is probably wise for hospitalists and geriatricians to collaborate on the optimal physician models for $\mathrm{HaH}$.

There may emerge a new specialty. Ticona and Schulman described a "home intensivist" with competencies including informatics of remote monitoring technology, leadership of multidisciplinary care teams, and the interpersonal skills required for compassionate end-of-life care. ${ }^{18}$ We prefer the term Home Hospitalist. Home Hospitalists would develop an enhanced understanding of the transitions of care and social determinants of health, and they would gain valuable knowledge about the social and environmental challenges many patients face after discharge from the hospital.

When this vision is realized, there will be enormous benefits to both $\mathrm{HaH}$ and Hospital Medicine. $\mathrm{HaH}$ could tap into a large and competent workforce to enhance its implementation and dissemination. Hospital Medicine would gain a new pathway for its providers and could develop new collaborative efforts with geriatric, internal, and family medicine.

Disclosures: Dr. Danielsson has nothing to disclose. Dr. Leff reports personal fees from Medically Home, other from Dispatch Health, other from Landmark Health, personal fees from Medibank, personal fees from Apple, personal fees from Health Affairs, other from Honor, personal fees from Institute for Healthcare Improvement, outside the submitted work; and American Academy of Home Care Medicine - member board of directors, voluntary.

Funding: Dr. Leff was supported in this work by a grant from The John A. Hartford Foundation.

\section{References}

1. Leff B, Montalto M. Home hospital-toward a tighter definition. J Am Geriatr Soc. 2004;52(12):2141. doi: 10.1111/j.1532-5415.2004.52579_1.x.

2. Leff B, Burton L, Mader SL, et al. Hospital at home: feasibility and outcomes of a program to provide hospital-level care at home for acutely ill older patients. Ann Intern Med. 2005;143(11):798-808. doi: 10.7326/0003-4819-14311-200512060-00008.

3. Brennan TA, Leape LL, Laird NM, et al. Incidence of adverse events and negli- 
gence in hospitalized patients. Results of the Harvard Medical Practice Study I. N Engl J Med. 1991;324(6):370-376. doi: 10.1056/NEJM199102073240604.

4. Creditor MC. Hazards of hospitalization of the elderly. Ann Intern Med. 1993:118(3):219-223. doi: 10.7326/0003-4819-118-3-199302010-00011.

5. Covinsky KE, Pierluissi E, Johnston CB. Hospitalization-associated disability: "She was probably able to ambulate, but I'm not sure". JAMA 2011;306(16):1782-1793. doi: 10.1001/jama.2011.1556.

6. Wald HL. The Geometry of Patient Safety: Horizontal and Vertical Approaches to the Hazards of Hospitalization. J Am Geriatr Soc. 2017;65(12):25592561. doi: 10.1111/jgs.15049

7. Shepperd S, Doll H, Angus RM, et al. Avoiding hospital admission through provision of hospital care at home: a systematic review and meta-analysis of individual patient data. CMAJ. 2009;180(2):175-182. doi: 10.1503/cmaj.081491.

8. Caplan GA, Sulaiman NS, Mangin N, et al. A meta-analysis of "Hospital in the Home". Med J Aust. 2012;197:512-519. doi: 10.5694/mja12.10480.

9. Shepperd S, lliffe S, Doll HA, et al. Admission avoidance hospital at home. Cochrane Database Syst Rev. 2016;9:CD007491. doi: 10.1002/14651858. CD007491.pub2.

10. Federman AD, Soones $T$, DeCherrie LV, Leff B, Siu AL. Association of a bundled hospital-at-home and 30-day postacute transitional care program with clinical outcomes and patient experiences. JAMA Intern Med. 2018;178(8):1033-1040. doi: 10.1001/jamainternmed.2018.2562.

11. Stein PD, Hull RD, Matta F, Willyerd GL. Modest response in translation to home management of deep venous thrombosis. Am J Med. 2010;123(12):1107-1113. doi: 10.1016/j.amjmed.2010.07.016.
12. Icahn School of Medicine at Mount Sinai. "HaH-Plus" (Hospital at Home Plus) Provider Focused Payment Model. https://aspe.hhs.gov/system/files/ pdf/255906/HaHPlusProviderFocusedPaymentModel.pdf. Accessed November 11, 2018.

13. Physician-Focused Payment Model Technical Advisory Committee. Report to the Secretary of Health and Human Services. Comments and Recommendation on "HaH-Plus (Hospital at Home Plus) Provider-Focused Payment Model. https://aspe.hhs.gov/system/files/pdf/255906/MtSinaiHAHReportSecretary.pdf. Accessed November 11, 2018.

14. The Secretary of Health and Human Services. Response to the Report to the Secretary of Health and Human Services. Comments and Recommendation on "HaH-Plus (Hospital at Home Plus) Provider-Focused Payment Model. https://downloads.cms.gov/files/cmmi/ptac-hhssecresponse-oct17-may18. pdf. Accessed November 11, 2018.

15. Montalto M. The 500-bed hospital that isn't there: the Victorian Department of Health review of the Hospital in the Home program. Med J Aust. 2010;193(10);598-601.

16. Hayashi J, Leff B. Geriatric Home-Based Medical Care. New York, NY: Springer Publishers; 2015.

17. Reckrey JM, Ornstein KA, Wajnberg A, Kopke MV, DeCherrie LV. Teaching home-based primary care. Home Healthc Now. 2017;35(10):561-565. doi: 10.1097/NHH.0000000000000621.

18. Ticona $L$, Schulman KA. Extreme home makeover - the role of intensive home health care. N Engl J Med. 2016;375(18):1707-1709. doi: 10.1056/NEJMp1608301. 